\title{
Análise da Variação Espacial do Parâmetro pH no Aquífero Bauru nos anos 2010, 2011 e 2012
}

Spatial variation analysis of pH parameter in the Bauru Aquifer from 2010 to 2012

Análisis de variación espacial del parámetro de pH en el Bauru Acuífero de 2010 a 2012

Sâmia Momesso Marques Mestranda do PPGEC, UNESP - Ilha Solteira/SP, Brasil. samia.momesso@gmail.com

César Gustavo da Rocha Lima Professor Doutor, UNESP - Ilha Solteira/SP, Brasil. etaugustus@hotmai.com 


\section{RESUMO}

A utilização dos recursos hídricos tem sido cada vez mais elevada, frente ao considerável crescimento populacional e desenvolvimento de cidades. A maior busca aos recursos hídricos tem atingido tanto as águas superficiais quanto as subterrâneas. Juntamente à elevada demanda, as exigências por águas de qualidade também têm aumentado. As principais fontes de poluentes estão associadas aos esgotos domésticos urbanos, aos efluentes industriais e às águas de escoamento superficial, que carreiam desde lixos de áreas urbanas, a agrotóxicos em áreas agrícolas. De forma geral, as fontes de poluição se propagam no meio, afetando além do local de sua fonte. Para avaliar a qualidade da água é necessário constante monitoramento, e para isso existem diversos parâmetros que podem ser tomados como base. Um importante parâmetro associado à qualidade de consumo da água, é o pH. Ele indica a medida dos íons hidrogênio e expressa a intensidade das condições ácidas ou alcalinas da água. Tendo intervalos permitidos para despejos em corpos hídricos e também para consumo humano. $O$ objetivo deste estudo foi avaliar a variabilidade do $\mathrm{pH}$ nas águas do Aquífero Bauru, ao longo dos anos 2010, 2011 e 2012. Foi realizada a caracterização do local de estudo, com o levantamento dos dados e localização dos pontos de coleta. Após esta etapa, foi feita análise estatística e geoestatística, para então desenvolver o mapeamento da área por período, tudo com o auxílio de programas computacionais. Os dados apresentaram homogeneidade e elevada consistência, comprovando a eficácia do método e se aproximando significativamente da realidade.

PALAVRAS-CHAVE: Água subterrânea. Semivariograma. Krigagem.

\section{ABSTRACT}

The water resources have been increasingly higher usage, as a result of considerable population growth and cities development. The search for water resources have been affect both superficial and underground water. Also appear demands for higher water qualities. The main source pollutants are associated with public sewerage, industrial effluents and runoff superficial, which bring garbage of urban centers, or even pesticides of agricultural areas. On the role, the source pollutant spread apart from the source. For evaluate the water quality is necessary constant monitoring, and for that exist many parameters. An important parameter linked to quality of drinking water is the $\mathrm{pH}$. It measures the hydrogen ions and express the intensity of acid and alkaline conditions. There are $\mathrm{pH}$ permitted values to discharge of water in water bodies and to water of human consumption. The aim of this study was evaluate the $\mathrm{pH}$ variability in Bauru Aquifer in the period from 2010 to 2012. It has been realized the local characterization, with data collection and location of the points. After this stage, it has been done the statistical and geostatistical analysis for then develop the area map. All this stages have been done with the aid of computer programs. The data presented homogeneity and high consistency, proving the method efficacy and showing results close to reality.

\section{KEYWORDS: Underground water. Semivariogram. Kriging}

\section{RESUMEN}

Los recursos hídricos han sido cada vez más utilizados, como resultado del considerable crecimiento de la población y el desarrollo de las ciudades. La búsqueda de recursos hídricos ha afectado tanto el agua superficial como subterránea. También aparecen demandas para más altas calidades de agua. Los principales contaminantes de origen están asociados con alcantarillado público, efluentes industriales y escorrentías superficiales, que traen basura de centros urbanos, o incluso pesticidas de áreas agrícolas. Para evaluar la calidad del agua es necesario monitoreo constante, y para ello existen muchos parámetros. Un parámetro importante relacionado con la calidad del agua potable es el pH, mide los iones de hidrógeno y expresa la intensidad de las condiciones ácidas y alcalinas. Existen valores de $\mathrm{pH}$ permitidos para la descarga de agua en cuerpos de agua y agua de consumo humano. El objetivo de este estudio fue evaluar la variabilidad del pH en el acuífero de Bauru en el período de 2010 a 2012. Se ha realizado la caracterización local, con recolección de datos y localización de los puntos. Después de esta etapa, se ha realizado el análisis estadístico y geoestadístico para luego desarrollar el mapa de área. Todas estas etapas se han hecho con la ayuda de programas informáticos. Los datos presentaron homogeneidad y alta consistencia, demostrando la eficacia del método y mostrando resultados cercanos a la realidad.

PALABRAS-CLAVE: Parámetro de calidad. Aguas subterráneas. Semivariograma. Krigagem 


\section{INTRODUÇÃO}

Ao longo da história da humanidade, as necessidades de uso da água foram se tornando crescentemente mais diversificadas e exigentes, em quantidade e qualidade (HÉLLER e PÁDUA, 2006). As populações cresceram quanto ao número de indivíduos e também em questões de área ocupada na superfície terrestre.

Quanto aos recursos hídricos, tanto superficiais quanto subterrâneos, as demandas têm crescido consideravelmente. Os usos são os mais variados, necessidades básicas humanas de consumo e higiene, até o uso em indústrias. De acordo com Héller e Pádua (2006), dentre os principais usos consuntivos podemos destacar o abastecimento doméstico e industrial, a irrigação e a aquicultura. Como uso não-consuntivo estão a geração de energia em hidroelétricas, a navegação, a recreação, a pesca e a diluição, ou seja, assimilação e afastamento de efluentes.

Segundo WHO (1996), com o advento da industrialização e o crescimento populacional, a gama de requisitos para a água aumentou juntamente com maiores exigências de água de alta qualidade. De forma geral, a poluição e a degradação da qualidade da água interferem diretamente seu uso, em qualquer escala ou região do mundo.

As fontes de poluição podem ser classificadas em atmosféricas, não atmosféricas, pontuais e difusas. De acordo com WHO (1996), a atmosfera prova ser a fonte mais abrangente de poluentes no meio ambiente global. Além disso, quantidades significantes de contaminantes são observadas na neve e gelo do Ártico e da Antártida. As fontes atmosféricas incluem: combustão de combustíveis fósseis para a geração de energia, queima destes combustíveis, fundição de minérios, movimentação do solo de regiões áridas ou agriculturas pela ação do vento e volatilização na agricultura. De forma geral, as fontes de poluição se propagam no meio, afetando além do local de sua fonte.

Quanto as águas, após sua utilização, deveriam sempre ser devolvidas, através dos efluentes, estando livres de dejetos e contaminantes que não se faziam presentes antes do uso, para que os ecossistemas sofram o mínimo possível de impactos ambientais. Apesar da importância de tratamentos eficazes isso nem sempre ocorre. Segundo Domingues (2006), as três maiores fontes de poluição da água são os esgotos domésticos urbanos, os efluentes industriais e as águas de escoamento superficial, que carreiam lixo das áreas urbanas e defensivos e fertilizantes agrícolas para os corpos de água.

Para avaliar a qualidade da água é necessário realizar o monitoramento constante da mesma, para isso, existem diversos parâmetros que servem como indicativos. Um importante parâmetro associado à qualidade de consumo da água é o Potencial Hidrogeniônico (pH). Ele é de considerável importância, pois influencia diversos processos biológicos e químicos (WHO, 1996).

$\mathrm{O} \mathrm{pH}$ da água indica a medida da atividade dos íons hidrogênio e expressa a intensidade de condições ácidas, $\mathrm{pH}<7$, ou alcalinas, $\mathrm{pH}>7$. As águas naturais tendem a apresentar valores de $\mathrm{pH}$ próximos a neutralidade (7), e os critérios de proteção à vida aquática fixam o pH entre 6 e 9. Segundo CETESB (2015), por influir em diversos equilíbrios químicos que ocorrem 
naturalmente ou em processos unitários de tratamento de águas, o pH é um parâmetro importante em muitos estudos no campo do saneamento ambiental.

Na legislação do Estado de São Paulo, é estabelecida faixa entre 5 e 9 para o lançamento direto nos corpos receptores (artigo 18 do Decreto 8.468/76) e entre 6 e 10 para o lançamento na rede pública seguida de estação de tratamento de esgotos (artigo19-A) (CETESB, 2015). Recomenda-se, pela Portaria no 2914 do Ministério da Saúde (BRASIL, 2011), que o pH da água num sistema de abastecimento, seja mantido na faixa de 6 a 9,5.

As técnicas geoestatísticas, com base na construção de semivariogramas, permitem a descrição da dependência espacial das propriedades estudadas (VIEIRA e LOMBARDI NETO, 1995).

O presente estudo teve como objetivo avaliar a variabilidade espacial do parâmetro pH nas águas do Aquífero Bauru, ao longo dos anos 2010, 2011 e 2012.

\section{METODOLOGIA}

\subsection{Caracterização do Local de Estudo}

O Aquífero Bauru está localizado a oeste do Estado de São Paulo, seu quadrante pode ser definido pelas coordenadas GMS $26^{\circ} 06^{\prime} 36^{\prime \prime}$ S e $23^{\circ} 06^{\prime} 36^{\prime \prime}$ W; $19^{\circ} 46^{\prime} 12^{\prime \prime}$ e $47^{\circ} 59^{\prime} 24^{\prime \prime}$. O estudo teve como base os dados técnicos disponíveis pela CETESB (2013), a qual realiza o monitoramento das águas do Aquífero Bauru a partir de 75 pontos de coleta (Figura 1). Tais pontos encontram-se distribuídos de forma assimétrica em 71 municípios distintos.

Para cada ponto de coleta no Aquífero são oferecidos dados do período de cheia (março) e do período de seca (setembro), ou seja, dois valores anuais. Para realizar a identificação de cada atributo foi incluído, subscrito, junto ao nome do parâmetro os números 01 ou 02 para março e setembro, respectivamente, seguido do ano de coleta. Por exemplo, $\mathrm{pH}_{(01-12)}$ refere-se ao dado de $\mathrm{pH}$ em março de 2012.

\subsection{Análise Estatística Geoestatística do pH}

Para cada parâmetro estudado foi efetuada a análise descritiva com o auxílio da estatística clássica, utilizando o SAS (SCHLOTZHAVER e LITTELL, 1997), onde foram obtidas as medidas estatísticas média, mediana, valores mínimo e máximo, desvio padrão, coeficiente de variação, curtose, assimetria e posteriormente realizada análise da distribuição de frequência.

De acordo com Pimentel-Gomes e Garcia (2002), a variabilidade de um atributo pode ser classificada segundo a magnitude de seu coeficiente de variação (CV), sendo baixo quando (CV $\leq 10 \%)$, médio $(10 \%<\mathrm{CV} \leq 20 \%)$, alto $(20 \%<\mathrm{CV} \leq 30 \%)$ e muito alto (CV > 30\%). Assim sendo, o CV indica, quando elevado, a heterogeneidade dos dados, e quando baixo é indicador de homogeneidade (FROGBROOK et al., 2002). 
Figura 1: Localização dos 75 pontos de coleta no Aquífero Bauru

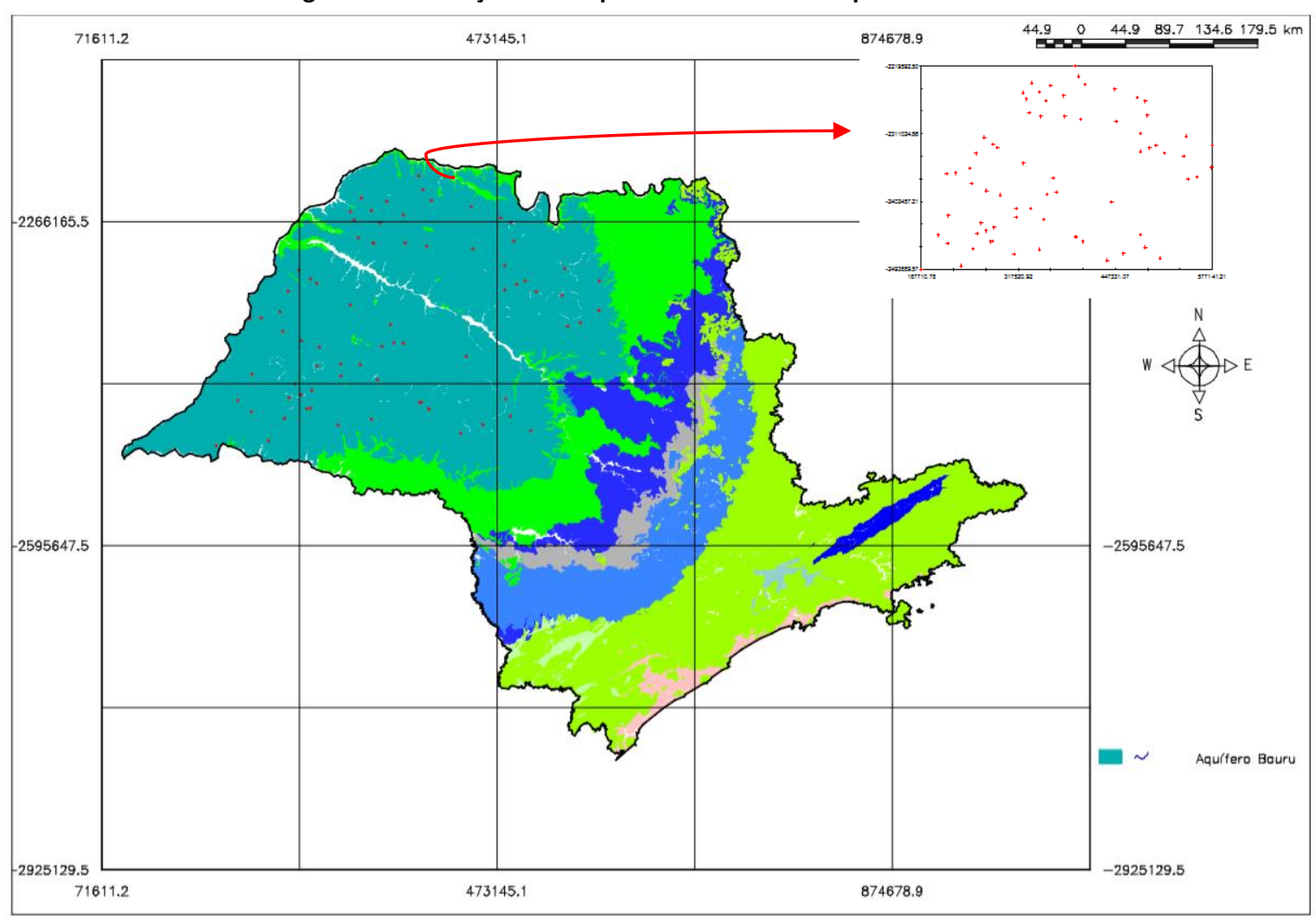

A partir da disposição espacial dos 75 pontos de coleta, dispôs-se uma malha irregular de dados para a análise geoestatística. Assim, foi avaliada a dependência espacial através da realização de semivariogramas, partindo das pressuposições da estacionaridade da hipótese intrínseca, pelo uso do pacote computacional Gamma Design Software GS+ 7.0 (ROBERTSON, 2004).

A análise da dependência espacial do $\mathrm{pH}$ foi avaliada por meio de ajustes de modelos matemáticos aos semivariogramas experimentais e a escolha do melhor modelo ajustado com base na pressuposição de estacionariedade da hipótese intrínseca (MATHERON, 1963) estimado por meio da expressão que segue (DA SILVA JUNIOR, 2013):

$$
\gamma(h)=\frac{1}{2 N(h)} \sum_{i=1}^{N(h)}\left[Z\left(x_{i}\right)-Z\left(x_{i}+h\right)\right]^{2}
$$

Onde: $N(h)$ é o número de pares experimentais de observações $Z\left(x_{i}\right)$ e $Z\left(x_{i}\right)+h$ separados por uma distância $\mathrm{h}$.

Os ajustes dos semivariogramas foram realizados em função de seus modelos (esférico, exponencial e gaussiano), partiram da seleção inicial dos princípios de: 1) A menor soma dos quadrados dos desvios (RSS); 2) O maior coeficiente de determinação $\left(r^{2}\right)$, e 3) O maior avaliador da dependência espacial (ADE).

Para obtenção do modelo representante final do ajuste realizou-se a validação cruzada, assim como para a definição do tamanho da vizinhança que proporcionou os melhores valores de 
krigagem. Para o parâmetro foi avaliado o efeito pepita puro (Co), o patamar $(\mathrm{Co}+\mathrm{C})$, e o alcance (Ao). A análise do avaliador de dependência espacial ( $A D E)$ foi calculada com base nos estudos desenvolvidos por Cambardella, Moorman e Novak (1994), modificado por Robertson (2004), conforme a expressão que segue:

$$
A D E=\left[C /\left(C+C_{O}\right)\right] * 100
$$

Onde: $A D E$ é o avaliador da dependência espacial; $C$ é a variância estrutural; $C+C_{O}$ é o patamar.

A interpretação proposta para o $A D E$ foi de que: a) $A D E<20 \%=$ variável espacial de dependência muito baixa; b) $20 \leq A D E<40 \%$ = dependência baixa; c) $40 \% \leq A D E<60 \%=$ dependência média; d) $60 \% \leq \mathrm{ADE}<80 \%$ = dependência alta; e) $80 \% \leq \mathrm{ADE} \leq 100 \%=$ dependência muito alta. Assim sendo, trabalhou-se na obtenção do número ideal de vizinhos, foram obtidos os mapas de interpretação e o detalhamento da variabilidade espacial dos atributos pesquisados por meio da interpolação por krigagem.

\section{RESULTADOS}

A análise descritiva realizada através da estatística básica segue indicada na Tabela 1:

Tabela 1: Medidas estatísticas descritivas

\begin{tabular}{cccccccccc}
\hline Período $^{(\text {a) }}$ & & \multicolumn{2}{c}{ Valor } & & $\begin{array}{c}\text { Desvio } \\
\text { Padrão }\end{array}$ & \multicolumn{3}{c}{ Coeficiente } & \multicolumn{3}{c}{$\begin{array}{c}\text { Probabilidade } \\
\text { (b) }\end{array}$} \\
& Média & Mínimo & Máximo & & Variação (\%) & Curtose & Assimetria & Pr<w & DF \\
\hline $01-10$ & 6,69 & 5,00 & 9,40 & 0,85 & 12,71 & 0,61 & 0,83 & - & IN \\
$02-10$ & 6,75 & 5,00 & 9,00 & 0,68 & 10,01 & 1,90 & 0,11 & - & IN \\
$01-11$ & 6,84 & 5,00 & 9,10 & 0,77 & 11,20 & 1,24 & 0,10 & - & IN \\
$02-11$ & 6,96 & 5,90 & 9,20 & 0,63 & 9,07 & 2,64 & 0,80 & - & IN \\
$01-12$ & 7,23 & 5,60 & 9,10 & 0,74 & 10,24 & 0,72 & 0,72 & - & IN \\
$02-12$ & 6,88 & 5,00 & 9,20 & 0,73 & 10,57 & 0,92 & 0,16 & - & IN
\end{tabular}

${ }^{\text {(a) } 01 ~ e ~} 02$ indicam respectivamente o primeiro e o segundo semestre dos seguintes anos: 2010, 2011 e 2012 ; ${ }^{\text {(b) }}$ DF = distribuição de frequência, sendo NO, TN, LN e IN respectivamente do tipo normal, tendendo à normal, lognormal; tendendo à lognormal e indefinido.

Com base nos dados estatísticos é possível observar que os valores de $\mathrm{pH}$ encontram-se entre o valor mínimo de 5,0 e máximo de 9,4. O valor mínimo está dento do padrão em relação ao valor permitido pelo Estado de São Paulo para lançamento de efluentes em corpos hídricos, porém para o critério de proteção de vida aquática ou para água de abastecimento ele necessitaria de uma correção de pH, devido a indicação de mínimo ser igual a 6,0. Quanto ao valor máximo, ele se encontra acima do permitido para lançamento em corpos hídricos $(9,0)$, mas dentro do padrão de captação do Estado de São Paulo $(10,0)$.

Os valores de Coeficiente de Variação, por estarem localizados entre $10 \%$ e $20 \%$, representam a homogeneidade dos dados de $\mathrm{pH}$ na área de estudo.

Na Tabela 2 estão indicados os parâmetros de ajustes adotados no Gamma Design Software GS+ 7.0 para a obtenção das melhores adequações do mapa de krigagem (Figura 2). 
(1)

(. - (3)

Tabela 2: Parâmetros dos semivariogramas e das validações cruzadas ajustados

\begin{tabular}{|c|c|c|c|c|c|c|c|c|c|c|}
\hline \multirow[t]{3}{*}{$\mathrm{pH}^{(\mathrm{a})}$} & \multicolumn{10}{|c|}{ Parâmetros do Ajuste } \\
\hline & \multirow[t]{2}{*}{ Modelo $^{(\mathbf{b})}$} & \multirow[t]{2}{*}{$\mathrm{C}_{0}$} & \multirow[t]{2}{*}{$\mathrm{C}_{0}+\mathrm{C}$} & \multirow[t]{2}{*}{$A_{0}(m)$} & \multirow[t]{2}{*}{$r^{2}$} & \multirow[t]{2}{*}{$S Q R^{(c)}$} & \multirow{2}{*}{$\frac{A D E^{(d)}}{\%}$} & \multicolumn{3}{|c|}{ Validação Cruzada } \\
\hline & & & & & & & & $a$ & $B$ & $\mathbf{R}$ \\
\hline 01- & $\operatorname{gau}_{15}^{14}$ & $1,220.10^{1}$ & $5,010.10^{1}$ & 51.441 & 0,829 & $2,690 \cdot 10^{-1}$ & 75,6 & $-0,150$ & 1,022 & 0,675 \\
\hline $\begin{array}{l}02- \\
01-\end{array}$ & $\begin{array}{l}\text { gau } \\
\operatorname{gau}^{13}\end{array}$ & $\begin{array}{l}1,300.10 \\
2,190.10^{-3}\end{array}$ & $1,958.10^{-2}$ & 42.435 & 0,718 & $\begin{array}{l}4,300 \cdot 10 \\
8,886 \cdot 10^{-5}\end{array}$ & $\begin{array}{l}96,6 \\
88,8\end{array}$ & $\begin{array}{l}0,9<0 \\
0,900\end{array}$ & $\begin{array}{l}0,863 \\
0,872\end{array}$ & $\begin{array}{l}0,038 \\
0,516\end{array}$ \\
\hline $02-$ & $\exp ^{15}$ & $1,000 \cdot 10^{-3}$ & $5,000 \cdot 10^{-1}$ & 84.300 & 0,892 & $1,210.10^{-2}$ & 99,8 & 1,500 & 0,783 & 0,397 \\
\hline $01-$ & $\exp ^{17}$ & $7,600 \cdot 10^{-2}$ & $5,850 \cdot 10^{-1}$ & 165.900 & 0,934 & $1,460.10^{-2}$ & 87,0 & -0.030 & 1,004 & 0,662 \\
\hline $02-$ & $e s f^{15}$ & $6,300 \cdot 10^{-2}$ & $5,340 \cdot 10^{-1}$ & 55.500 & 0,688 & $5,860 \cdot 10^{-2}$ & 88,2 & $-0,370$ & 0,177 & 0,583 \\
\hline
\end{tabular}

(a) 01 e 02 referem-se ao primeiro e segundo semestre; 10, 11 e 12 referem-se aos anos 2010, 2011 e 2012; ${ }^{(b)}$ Ajuste semivariográfico, sendo: gau = gaussiano, exp = exponencial, esf = esférico (seguidos dos respectivos valores de pares do 10 lag); epp = efeito pepita puro; TRQ = Transformação Raíz Quadrada; ${ }^{(c)}$ SQR = Soma dos Quadrados dos Resíduos; ${ }^{(d)} \mathrm{ADE}=$ Avaliador da Dependência Espacial.

No período analisado foram observados apreciáveis ajustes dos valores de $\mathrm{pH}$, atestados pelos parâmetros dos semivariogramas. Foram verificados ajustes do tipo gaussiano (01-10; 02-10; 01-11), exponencial (02-11; 01-12) e esférico (02-12), com valores de alcance entre $52.435 \mathrm{~m}$ e $165.900 \mathrm{~m}$, valores consideravelmente altos devido a extensão da área de estudo.

Além disso, o coeficiente de determinação espacial $\left(r^{2}\right)$ variou de 0,717 a 0,934 e os avaliadores da dependência espacial (ADE) entre alto e muito alto. Os valores de $R$ da validação cruzada também foram elevados.

De acordo com estudo desenvolvido por Cambardella, Moorman e Novak (1994), as variáveis $\mathrm{pH}, \mathrm{Mg}^{2+}$, saturação por bases e $\mathrm{Cu}$ apresentam forte dependência espacial; as demais variáveis em seu estudo apresentaram moderada dependência. Isso demostra que os semivariogramas explicam a maior parte da variância dos dados (DE OLIVEIRA MACHADO et al., 2007).

Em estudo realizado por Coluna, Dias e Pinheiro (2007), foi observado o comportamento semelhante da variável $\mathrm{pH}$ ao longo do período de cheia estudado, com valores próximos à neutralidade (7). Para o período de seca, houve um aumento de $\mathrm{pH}$ em um ponto de coleta, possivelmente influenciado por uma estrada próxima ao local. Se tivessem sido gerados os mapas de krigagem, seria possível identificar, de forma mais precisa, a localização da área onde o pH sofreu alteração.

Vários autores, como Oliveira et al. (1999) e Bottega et al. (2012), têm estudado a variabilidade espacial de propriedades químicas e físicas dos solos, demonstrando a dependência espacial dos atributos. Porém, estudos que apliquem esta metodologia para águas não é tão frequente e pode apresentar resultados tão apreciáveis quanto nos solos. 


\section{Fórum Ambiental \\ da rilta ipalista}

Figura 2 - Mapa de krigagem da variabilidade do pH no Aquífero Bauru

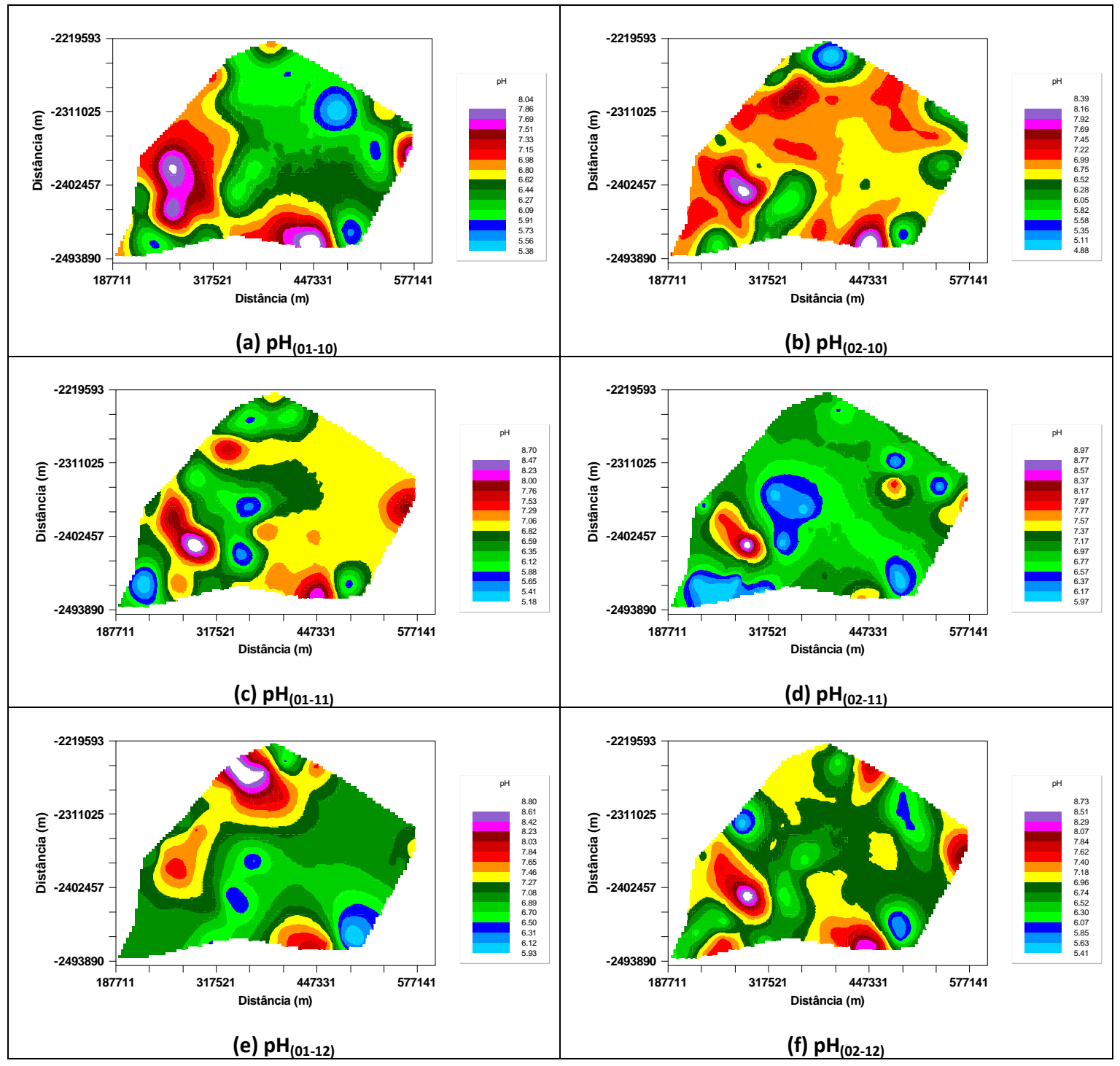

\section{CONCLUSÕES}

Quanto as estatísticas geradas, não é possível associar, sem análise prévia, que os valores de pH que se encontram superiores ao máximo permitido para efluentes no Estado de São Paulo, estão associados a efluentes lançados em corpos hídricos. As causas podem ser naturais, como o tipo de geologia da região, ou mesmo contaminação atmosférica transferida para águas subterrâneas por meio da absorção das águas de chuvas ácidas. Isto seria possível, pois o Aquífero Bauru é um aquífero superficial.

Em relação a utilização da água do Aquífero Bauru para abastecimento, seria necessário apenas se atentar aos valores mínimos, pois em alguns pontos a acidez está acima do permitido para o abastecimento humano, segundo a recomendação do Ministério da Saúde.

É possível observar nos mapas que as regiões de máxima e mínima mantiveram uma constância, apresentando algumas alterações pontuais, que poderiam ser avaliadas 
separadamente, considerando as relações às atividades locais, como centros urbanos, centros industriais ou agricultura.

Os dados do parâmetro pH apresentaram elevada consistência e homogeneidade, podendo ser considerados aproximações da realidade do local, e confirmando a eficácia do método aplicado.

\section{AGRADECIMENTO}

CAPES pelo apoio financeiro, através da concessão da bolsa de mestrado.

\section{REFERÊNCIAS BIBLIOGRÁFICAS}

BOtTEGA, E. L.; QUIROZ, D. M.; DE CARVALho PINTO, F. D. A.; DE SOUZA, C. M. A. Variabilidade espacial de atributos do solo em sistema de semeadura direta com rotação de culturas no cerrado brasileiro, Revista Ciência Agronômica, v. 44, n. 1, p. 1-9, 2012.

CAMBARDELLA, C. A.; MOORMAN, T. B.; NOVAK, J. M. Field-scale variability of soil properties in central lowa soils. Soil Science Society of American Journal, Madison, v. 58, n. 5, p. 1501-1511, 1994.

COLUNA, N. M. E.; DIAS, H. C. T.; PINHEIRO, J. A. C. Análise temporal e espacial da qualidade da água na bacia hidrográfica do Zerede, Timóteo-MG, 2007.

COSTA, P. S. F.; MOREIRA, A. A. M.; CRUZ, E. F.; NEIVA, J. A. F.; INÁCIO, R. D. Avaliação da Qualidade das Águas Superficiais da sub-bacia do Rio Capivari no Município de Chapada do Norte-MG. Caderno Geografia, v. 26, n. 1, 2016.

CETESB. COMPANHIA DE TECNOLOGIA DE SANEAMENTO AMBIENTAL. Qualidade das Águas Subterrâneas no Estado de São Paulo. Série Relatórios. São Paulo, 2013.

CETESB. COMPANHIA DE TECNOLOGIA DE SANEAMENTO AMBIENTAL. Qualidade das águas superficiais do Estado de São Paulo. Apêndice D - Significado Ambiental e Sanitário das Variáveis de Qualidade. São Paulo, 2015.

DA SILVA JUNIOR, J. F.; GENER, T. P.; CAMARGO, L. A.; JÚNIOR, J. M. Métodos geoestatísticos na modelagem espacial do diâmetro médio do cristal da goethita. Revista Brasileira de Engenharia Agrícola e Ambiental, v. 17, n. 11, p. 1127-1134, 2013.

DE OLIVEIRA MACHADO, L; QUINTÃO LANA, Â. M.; QUINTÃO LANA, r. M.; GUIMARÃES, E. C.; FERREIRA, C. V. Variabilidade espacial de atributos químicos do solo em áreas sob sistema plantio convencional. Revista Brasileira de Ciência do Solo, v. 31, n. 3, 2007.

FROGBROOK, Z. L.; OLIVER, M. A.; SALAHI, M.; ELLIS, R. H. Exploring the spatial relations between cereal yield and soil chemical properties and the implications for sampling. Soil Use and Management, Oxon, v. 18, n. 1, p. 1-9, 2002.

HÉLLER, Léo; DE PÁDUA, Valter Lúcio. Abastecimento de água para consumo humano. Editora UFMG, 2006.

MATHERON, G. Principles of geostatistics. Economic Geology, v. 58, p. 1246-1266, 1963.

OLIVEIRA, J. J., CHAVES, L. H. G.; QUEIROZ, J. E.; LUNA, J. G. Variabilidade espacial de propriedades químicas em um solo sonilo-sódico, Revista Brasileira de Ciência do Solo, v. 23, n. 4, p. 783-789, 1999.

PIMENTEL-GOMEZ, F.; GARCIA, C. H. Estatística aplicada a experimentos agronômicos e florestais: exposição com exemplos e orientações para uso de aplicativos. Piracicaba: Fealq, 2002. 309 p. 
ROBERTSON, G.P. GS+: geostatistics for environmental sciences. 7. ed. Michigan: Gamma Desing Software, 2004. $159 \mathrm{p}$.

SCHLOTZHAVER, S. D.; LITTELL, R. C. SAS system for elementary statical analysis. 2. ed. Cary: SAS, 1997. 441 p.

SHAPIRO, S. S.; WILK, M. B. An analysys of variance test for normality: complete samples. Biometrika, London, v. 52, n. 3/4, p. 591-611, 1965.

VIEIRA, S. R.; LOMBARDI NETO, F. Variabilidade espacial do potencial de erosão das chuvas do Estado de São Paulo, Bragantia, v. 54, p. 405-412, 1995.

WHO. World Health Organization. Water Quality Assessments - A Guide to Use of Biota, Sediments and Water in Environmental Monitoring. London, England. 2ed., p.651, 1996. 